\title{
Evaluating the Information Power Grid using the NAS Grid Benchmarks
}

\author{
Rob F. Van der Wijngaart*and Michael A. Frumkin \\ NASA Advanced Supercomputing Division \\ M/S T27A-1, NASA Ames Research Center \\ Moffett Field, CA 94035-1000 \\ \{wijngaar,frumkin\}@nas.nasa.gov
}

\begin{abstract}
The NAS Grid Benchmarks (NGB) are a collection of synthetic distributed applications designed to rate the performance and functionality of computational grids. We compare several implementations of the $N G B$ to determine programmability and efficiency of NASA's Information Power Grid (IPG), whose services are mostly based on the Globus Toolkit. We report on the overheads involved in porting existing NGB reference implementations to the IPG. $N o$ changes were made to the component tasks of the NGB.
\end{abstract}

\section{Introduction}

As computational grids are gaining more acceptance and prominence, tools are reguired to determine which components of grids function well and which require improvement. To do this in a systematic way, a standard rating mechanism must be developed, i.e. grid benchmarks. Our approach is to develop such benchmarks primarily to serve grid users, since increased application programmer productivity and application performance are the main goals of computational grids. Consequently, we have focused on characterizing actual distributed applications that are suitable for execution on grids. The outcome of that work, the first pubilicly avaīab̄le grid benchmark suite, was reieased under the name NAS Grid Benchmarks (NGB), whose precise specification is described in [7]. The motivation, background and early experiences with NGB are reported in [3], along with a brief description of a reference implementation in Java.

In this paper we discuss two implementations of NGB on NASA's production computational grid called the Information Power Grid (IPG), and compare that to the earlier serial implementation. The performance results are reason for

\footnotetext{
${ }^{*}$ Computer Sciences Corporation
}

guarded optimism that IPG may be beneficial for NASA's application workload, but programmability and reliability can still be improved.

The rest of this paper is structured as follows. In Section 2 we briefly review the NGB, including the non-IPG reference implementations. We also discuss some other grid benchmarking and monitoring projects. In Section 3 we describe the software and hardware infrastructure of IPG relevant to our experiments. In Section 4 we give important details of our actual IPG NGB implementations. We discuss performance and programmability results in Section 5 .

Some concluding remarks about the current state of the IPG, as well as recommendations for enhancement, are presented in Section 6.

\section{Background}

The NGE capture a subset of distributed applications that can be cast in the form of data flow graphs (DFGs). Such

\section{Embarrassingly Distributed (ED)}

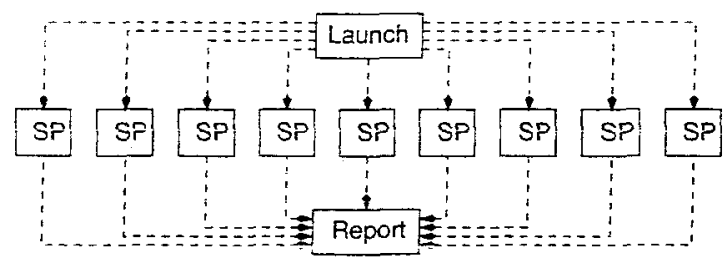

\section{Figure 1. Data flow graph representing ED} NAS Grid Benchmark.

applications are among the simplest in terms of software in frastructure required, and hence they should be represented in a basic grid benchmarking suite. The DFGs for these benchmarks, named Embarrassingly Distributed (ED), Helical Chain (HC), Visualization Pipe (VP), and Mixed Bag (MB), are depicted in Figures 1 and 2. The nodes of the 
Helical Chain $(\mathrm{HC})$

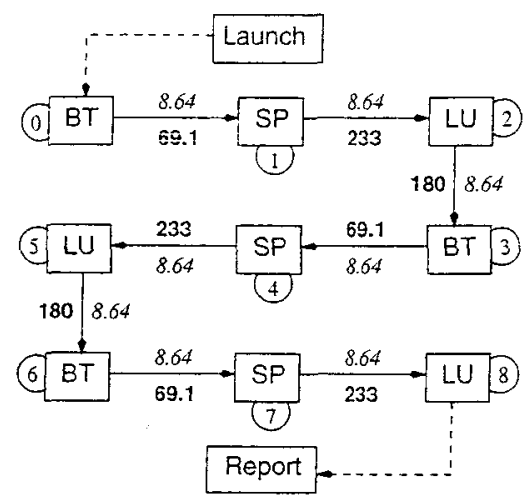

Visualization Pipe (VP)

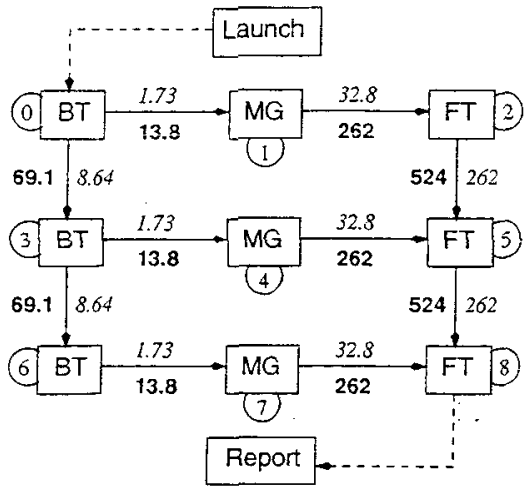

Mixed Bag (MB)

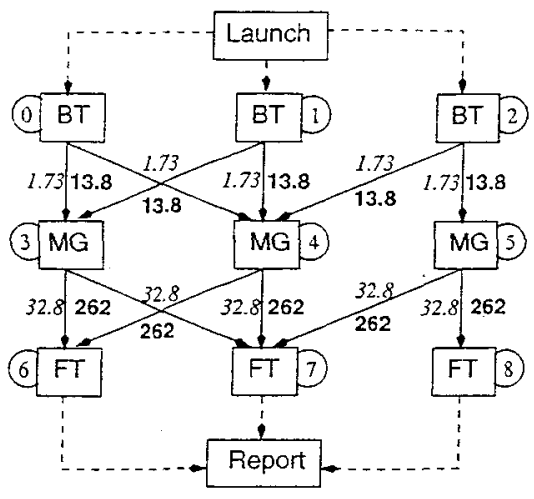

Figure 2. Data flow graphs representing HC, VP, and MB NAS Grid Benchmarks; numbers in italics and bold indicate thousands of words communicated between tasks for Classes $S$ and $W$, respectively. Tasks are numbered according to the labels in the semi-circles.

graphs, indicated by the rectangular boxes, are computationail tasks. Dashed arrows indicate controi flow between the tasks, and solid arrows indicate data as well as control flow. Communication volumes between tasks are indicated in Figure 2. Launch and Report do little work; they initiate execution of the task graph and collect performance and verification results, respectively. The other computational tasks, SP, BT, LU, MG, and FT, have some internal structure, see Figure 3 in Section 4. They are derived from the NAS Parallel Benchmarks (NPB) and involve computations on sizable multi-dimensional arrays. When the arrays on connected nodes are of the same size, no data transformation needs to take place, but if a node has more than one input arcs and/or if the arrays on connected nodes are of different size, an additional computation needs to occur to merge and/or interpolate data. This computation is carried out by a function called Mesh Filter [7]. The NGB are parameterized and can be run for different array sizes, which are usually referred to as Classes. In this study we use the two smallest Classes, named $\mathrm{S}$ and $\mathrm{W}$.

ED, HC, VP, and MB highlight different aspects of computational grids. ED requires vitually no communication, and all the tasks within the graph can be executed independently. It tests basic functionality of grids and does not tax their communication performance; but it does allow us to measure the cost of remote task creation. $\mathrm{HC}$ is totally sequential at the graph level. Hence, any time spent on communicating data between graph nodes is fully exposed and will show up in performance results. VP requires specifying concurrent execution of tasks in a DFG with nontrivial dependencies. It allows pipelining and overlapping communication times with computational work. $\mathrm{MB}$ is similar to $\mathrm{VP}$, but the nodes of the graph all have different amounts of computational work.

Other projects in the area of grid benchmarking and evaluation have thus far mainly concentrated on monitoring health and status of particular grid components. Examples are the Globus Heart Beat Monitor [10] (part of the Globus Toolkit [11]), the Network Weather Service [8], and Wren [9]. Recently, some efforts were made to determine which rcalistic applications and application classes qualify as potential grid benchmark examples $[6,12]$, but no final selections have yet been made. The GRASP project [2] provides a collection of low level probes that mostly measure the network capabilities of grids. It distinguishes itself from most other grid measurement projects in the sense that the probes are formulated and implemented as complete grid applications, including authentication, validation, and resource verification.

\section{Information Power Grid}

The Information Power Grid [5] was conceived to unify NASA's many and geographically dispersed computational resources. It contains a number of tools and components in its layered architecture, for work flow creation and management, scheduling, etc. At present much of the middleware is provided by the Globus Toolkit, version 2.4.2 [11], and this is the software that we use for implementing NGB on the IPG. In our experiments we also investigate the file transfer properties of two protocols that are not part of GT2.4, but that can be used with it: GridFTP, based on gsincftp tools (version 3.0.6), and gsiscp version 3.4. GridFTP, gsiscp, and Globus all use the same authentication method (Grid Security Infrastructure GSI).

Systems that are currently part of the IPG include four 
SGI Origin $2000(\mathrm{O} 2 \mathrm{~K})$ and two Origin 3000 (O3K) systems at NASA Ames in California, a PC Cluster and an O2K system at NASA Glenn in Ohio, and two O2K and one O3K systems at NASA Langley in Virginia [13]. For this study we use just the Origin systems at Ames.

\section{Implementations}

We describe two Globus implementations of NGB. The first, called NGBIPG-Ksh, uses Korn shell scripts to launch the tasks in the DFGs and to control data transfers. It is a direct extension of the serial NGB reference implementation using a single local file system, available from NAS as part of the GridNPB3.0 package [14]. We will refer to this serial implementation as SHF-Ksh. The second Globus implementation, called NGBIPG-Jav, uses Java code to communicate directly with GRAM [11] and GridFTP clients. Both Globus implementations use Fortran executables to do the computational work. At present these executables are serial themselves, but several can run simultaneously, depending on the parallelism present in the NGB DFGs.

Most of the Origin systems at NAS employ schedulers that act as the default Globus jobmanagers. To prevent jobs from getting stalled in queues we specify the fork jobmanager for all tasks. This is possible because all submitted jobs are of short duration and use minimal machine resources. Hence, they can run in machine partitions usually dedicated to interactive debugging and administrative operations. Once the larger NGB Classes are run, however, individual NGB tasks will require significantly more machine resources, and hence must be queued. Since HC, $V P$, and $M B$ feature denendencies between tasks, independent scheduling of tasks would be deleterious to IPG performance, and an approach must be taken that submits an entire NGB task graph-including all dependencies-to a grid scheduler, for example as it is done in the Grid Navigation System, [4], or in GRID Superscalar [1].

\subsection{NGBIPG Korn shell implementation}

To express the concurrency of ED, VP, and MB we adopt an asynehronous model in which the nodes of the DFGs are issued independently at the NGB launch, using the globusrun command with the batch flag "-b." The nodes poll their local file systems for the presence and completeness of the required input files (if any) through the use of semaphores (which are zero byte files themselves). After all nodes are started, the launch script periodically queries their completion using globus-job-status. To avoid using too many machine resources, the launch and node scripts poll only once a second.

The structure of the DFG nodes is indicated in Figure 3. Although $\mathrm{HC}$ is serial in nature, it can also be implemented

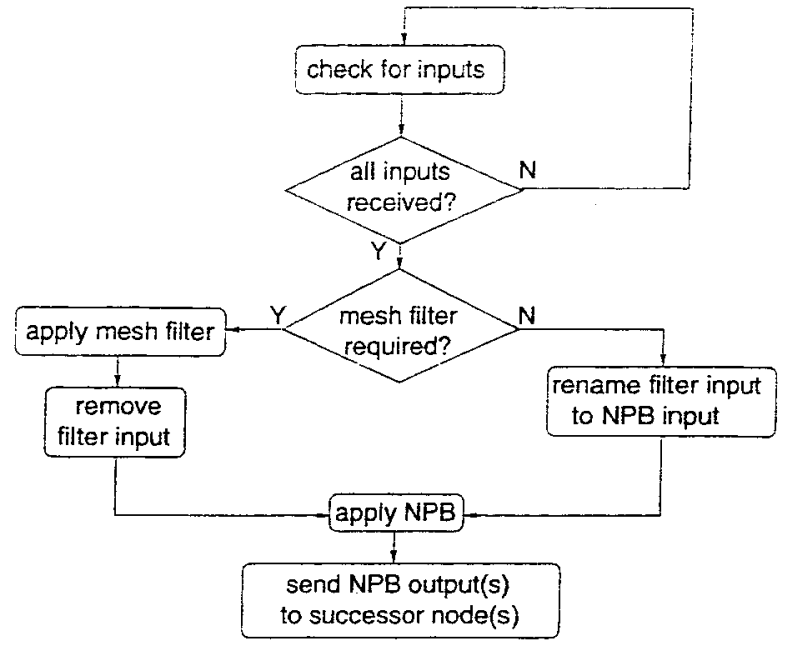

Figure 3. Flow chart of single node of Data Flow Graph.

using the asynchronous model; the nodes must run in sequence, but can be started asynchronously. Hence, we provide two versions of $\mathrm{HC}$, one synchronous, the other asynchronous. In the synchronous version, which is the most natural way of transforming the SHF-Ksh version for execution on the grid, the nodes are launched in blocking mode (i.e. using globus run in "non-batch mode). Only when the remote execution request returns is the next node launched. The following sequence describes the actions of the launch process. Run node $n$ on host $n$; transfer output file from host $n$ to host $n+1$; run node $n+1$ on host $n+1$. Note that this requires iuidu-party copying if host $n$ and host $n+1$ are different from each other and from the host running the launch process. Other than synchronous HC, all NGBIPGKsh benchmarks allow the choice between GridFTP and gsiscp to transfer files.

\subsection{NGBIPG Java implementation}

NGBIPG-Jav implementation uses GramJob and GridFTPClient Classes of the cog-1.1 package to access the GRAM and GridFTP servers. The actual access to the SHF-Ksh executables was accomplished by automatic creating shell scripts and coping them to the systems where the executables were invoked. The transfer of files was accomplished using the transfer method of the GridFTPClient class and execution of the scripts was accomplished using the request method of the GramJob class. Time required for creation of the shell scripts and their transfers to the grid machines was included in the turnaround time of the benchmarks.

For each NGB task we have to copy the following files: 
the shell script which executes the mesh filter and the actual NPB task, the final report of the task, each of the resulting data files, and the semaphore indicating that the result is ready. We also have to execute the chmod command on the remote system to allow execution of the script. Overall, it translates into $2 *(1+$ task output degree $)$ accesses to the GridFTP clients and two GramJob requests for each graph node, where task output degree equals the number of output files. We estimate that our code adds $1-2 \%$ overhead to the actual benchmark turnaround time in the form of time spent to write scripts and to poll internal job submission threads.

The submission of the NGB tasks was performed in a data flow manner. In other words, a task was submitted for execution on a statically specified host as soon as all its predecessors had finished their work and transferred the necessary files to the target machine. For ED it translates into simultaneous submission of all tasks; for HC into synchronous submission described in the previous subsection; and for VP and MB into submission of all tasks that were dynamically determined to be ready.

\subsection{Common implementation problems}

We identified the following issues in the development of the IPG versions of NGB, independent of the API chosen.

Preliminaries:

- Gridmap files containing IPG user information need to be updated on all grid machines by various system administrators before a proxy can be used there. The procedure is not transparent to the user. Messages from various administrators are confusing.

- Globus jobs can only be started from systems on which the user has obtained a proxy. This makes access to the grid asymmetrical, and the user has to remember where the proxy was obtained.

- It is possible to obtain a proxy on a host by copying the gridmap file from a machine on which an earlier proxy was obtained. This constitutes a security breach.

File transfer:

- GridFTP

1. Does not allow third-party copying of files.

2. Does not allow the renaming of the target file in the gsincetp command line interface (but does allow it in the Java API).

3. Does not retain the access permissions on files at the destination. For executables this necessitates issuing a "Chmod" command after the file transfer completes, with the concomitant overhead of a remote execution.
4. Will clobber a file that is copied onto itself, while no mechanism to avoid clobbering is provided.

Elimination of the first and second GridFTP restrictions is highly desirable for flexible programming. Third-party copying can always be accomplished through two two-party copies, but this is costly and usually leads to sequential bottlenecks. Target file renaming is useful, for example, in the semaphore technique used in NGBIPG-Ksh to signal successor nodes in the DFG that inputs have arrived. If a semaphore of the same name must exist on the sending host, it will not be possible to determine whether sending and receiving host share the same file system (as is the case for a number of hosts at NAS). Hence, a file transfer that is intended to be a move (destroy the copy at the source if sender and receiver use different file systems) cannot be safely implemented. Item 4 is especially harmful, as for the user it is often difficult or costly to determine whether two files on different hosts are actually the same. Safety measures to avoid accidental destruction of files are cumbersome to implement.

- globus_URL_COPY

1. Suffers from most of the ills that GridFTP does.

2. Requires knowledge of absolute paths of files on the various hosts on which grid users have accounts, since there is no concept of user home directories. This severely inhibits transparency of grid use and makes applications non-portable.

- gsiscp

1. This file transfer protocol has all the properties desirable for convenient programming (target file renaming, no clobbering, persistence of permissions, third-party copying), but is reported to be slower than GridFTP and globus_URL_copy (however, see Section 5 for a comparison).

\section{Execution environment:}

- The exit status of a remotely executing command is not available to the globusrun command that launched it. It has to be stored in a file and transferred to the place from which the globusrun command was issued.

- If the fork jobmanager is used to launch a remote script, the user's shell resources files are not sourced, which means that no path will be available, except that to globus commands through the \$GLOBUS LOCATION environment variable. If the batch scheduler jobmanager (Portable Batch System at NAS) is selected, resource files will get sourced. 
This means that jobs may succeed or fail, depending on what jobmanager is selected. A solution is always to source resource files in scripts executing through globusrun.

\section{Results}

We present performance results for the following experiments and configurations. To ensure repeatability no grid schedulers were used; we explicitly prescribe the machines on which the tasks are to be run. The machines used in our experiments are listed in Table 1.

Configurations boyd and dean consist of just those machines, respectively. Configuration $d u o$ consists of dean and boyd. Configuration mix consists of dean, boyd, alan, dean, joe, grace, dean, harv, and boyd. Tasks in the DFGs are mapped round robin to the sequence of machines in these configurations.

The turnaround time does not include deployment (copying) of executables; it does include deployment of scripts and the verification.

\subsection{Korn Shell}

In the experiments reported in Table 2 we always used gsiscp to copy semaphore files between hosts (GridFTP was not satisfactory, since its command line interface does not allow file renaming). An alternative to copying is to create semaphore files directly on remote systems by issuing touch commands through globusrun. However, in experiments with $\mathrm{HC}$ on configuration duo, the touch semaphore approach generally showed significantly poorer perfurmance than gsiscp, as weil as much greater vanability. For instance, 28 interleaved runs of HC Class S with both approaches showed average turnaround times that were 3.9 seconds longer for touch than for gsiscp. And whereas variability with gs iscp was within $17 \%$, touch produced differences of up to $57 \%$.

In our first NGBIPG-Ksh implementation, which did not yet include $\mathrm{MB}$, we did not provide methods to determine which hosts' file systems were cross-mounted and which were not. Consequently, some redundant copving (overwriting) of files occurred in the gsiscp HC and VP experiments on configuration mix. We did not obtain performance results for the GridFTP versions of $\mathrm{VP}$ and $\mathrm{HC}$ on configuration mix, since file clobbering occurred on the cross-mounted file systems ${ }^{\mathrm{I}}$.

\footnotetext{
'Once we had implemented $M B$ and supplied it with a mechanism to detect file systems shared by hosts, the IPG had changed and all configurations shared the same file system. Thus, in the duo and mix configurations of MB no redundant copying of files ever occurred, and accidental clobbering of files could be avoided. We did not rerun all other benchmarks using the same technique, because then we would not have been able to test the file transfer properties of $g s i s c p$ and GridFTP.
}

As expected, in the single-system configuration experiments, dean performed significantly better than boyd, since dean has much faster processors. Moreover, ED, which has the highest level of concurrency, quickly saturates the CPUs available to the fork jobmanager on boyd, whereas dean, which has many more processors, can accommodate all nine tasks simultaneously without performance deterioration. The reason why ED Class $S$ does not benefit as much from the faster processors as does Class $\mathrm{W}$ is that its performance is dominated by remote process creation and polling for results. This is demonstrated by comparing the IPG and non-IPG (10C) performance results of HC Class $S$; virtually all execution time is spent on process management in the IPG implementation. The effect of saturation is highlighted by the ED Class W experiments on configuration duo. Even though performance of ED is determined, in principle, by the slowest system in the configuration (duo utilizes both boyd and dean), turnaround time on duo is less than half that on boyd.

While $\mathrm{HC}$ is synchronous in nature, the asynchronous implementation always runs fastest. The reason is that remote process creation can be overlapped with computation, which the synchronous version does not allow.

The combined results of the $\mathrm{HC}$ and VP runs show that gs iscp is significantly faster than GridFTP on our testbed. When files are shared by tasks on the same host, we never copy, which explains why the gsi scp and GridFTP results on configurations boyd and dean are not affected by the file transfer protocol. However, on configuration duo, where two hosts with different file systems are used, VP with gsiscp is 19 seconds faster than with GridFTP for both benchmark classes. For HC, which cannot hide transmis-

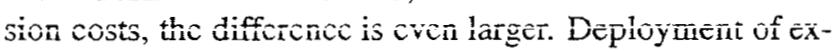
ecutables and scripts is not reported in Table 2, since it takes place before the actual job launch. However, we did measure these file copy speeds, and for HC and VP, GridFTP was between four and six times slower than gsiscp. For $E D$ the difference was a factor of three to four.

\subsection{Java}

Turnaround times of the NCBRIPG-Jav imnlementation are presented in Table 3. As was mentioned in Section 4, we perform $2 *(1+$ task output degree $)$ accesses to the GridFTP clients and make two GramJob requests within each graph node (Because of the shared file system on some machines, not all GridFTP calls actually result in a file copy.) For Class W of HC and VP we estimate that about $2 / 3$ of the turnaround time is spent accessing and delivering these IPG services, and $1 / 3$ of the time for the actual processing. Access and use of the services mostly involves network and memory subsystems, which blunts the raw processor speed advantage of dean. Otherwise, the same arguments used to 
Table 1. Grid Machines. The file systems of boyd, alan, joe, grace, and harv are cross-mounted, while dean has its own file system.

\begin{tabular}{|l||c|c|c|c|}
\hline \hline Name & Clock Rate $(\mathrm{MHz})$ & Architecture & Number of Processors & Processor Type \\
\hline boyd & 250 & O2K & 16 & MIPS R10000 \\
alan & 250 & O2K & 32 & MIPS R10000 \\
grace & 250 & O2K & 128 & MIPS R10000 \\
joe & 400 & O2K & 128 & MIPS R12000 \\
harv & 400 & O3K & 512 & MIPS R12000 \\
dean & 600 & O3K & 1024 & MIPS R14000 \\
\hline \hline
\end{tabular}

Table 2. Turnaround times (sec) for IPG (NGBIPG) and non-IPG (SHF) implementations using Korn shell scripts. Here conf indicates configuration, loc refers to running all tasks locally from a single script (SHF-Ksh). HC gsiscp-sync refers to the explicitly synchronous version. MB: see footnote on p.5.

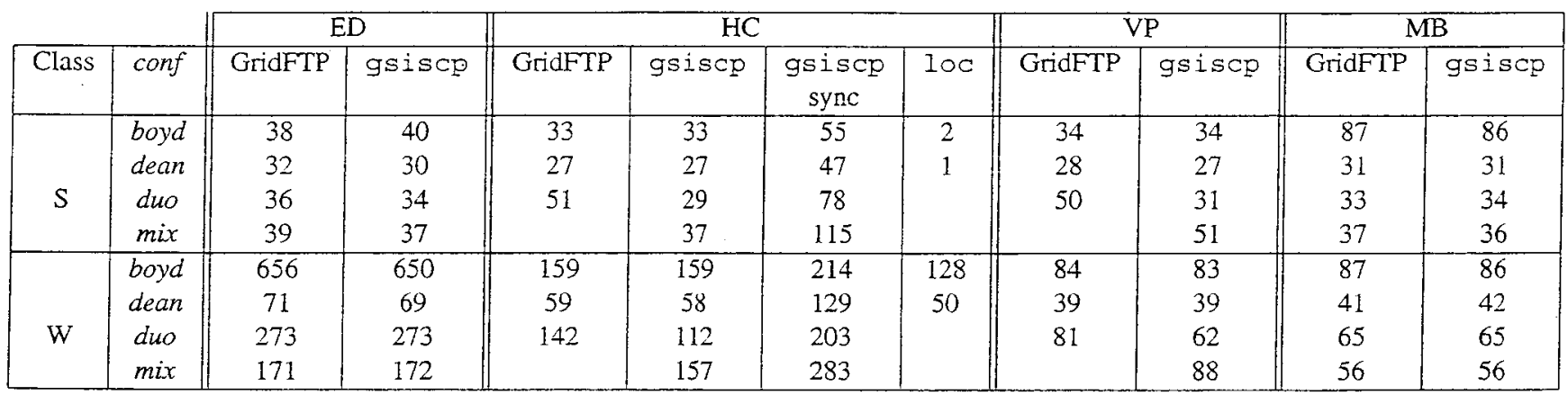

explain the NGBIPG-Ksh turnaround times are valid.

Table 3. Turnaround times (sec) for IPG (NGBIPG) implementations using Java. Here conf indicates configuration.

\begin{tabular}{|c|c|c|c|c|c|}
\hline Class & conf & ED & HC & VP & MB \\
\hline \multirow{5}{*}{$S$} & boyd & 104 & 251 & 205 & 165 \\
& dean & 83 & 242 & 176 & 117 \\
& duo & 101 & 255 & 192 & 139 \\
& mix & 93 & 271 & 200 & 183 \\
\hline \multirow{5}{*}{ W } & boyd & 660 & 320 & 243 & 228 \\
& dean & 132 & 247 & 179 & 165 \\
& duo & 404 & 296 & 231 & 203 \\
& mix & 230 & 346 & 214 & 204 \\
\hline
\end{tabular}

We have to be careful when interpreting the performance results, because a number of factors were beyond our control and not all could be measured independently. These include actual machine load, network traffic stability, and interference with other IPG users, setup of the IPG ser- vices (timeout, polling intervals etc.). To reduce influence of these random factors we repeat our experiments multiple times and report the best results (shortest turnaround times). Variation of turnaround times among runs reported in Tables 2 and 3 was within 20\%. Also, a number of our experiments failed because of IPG authentication failure. In these cases we just repeat the experiment with a 10 minutes delay.

Volatility of the performance of the grid machines is the major factor which can distort the performance of the IPG benchmarks. To gauge performance volatility we used the non-IPG Java version of the NGB [14] and monitored grid machines during some of our experiments. The monitoring results using ED.S, depicted in Figure 4, show that performance of the grid machines usually is within $20 \%$ of the average when all machines are up and the benchmark servers are properly installed.

\section{Conclusions}

It is important to recognize that our performance experiments are not exhaustive, and are focused on determining 


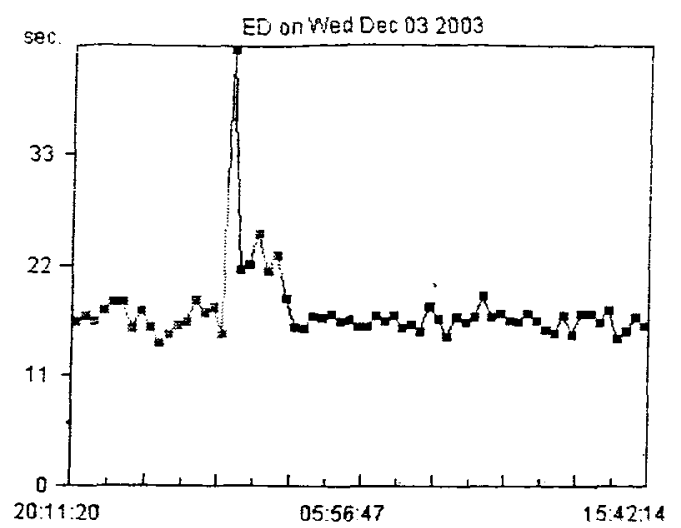

Figure 4. Typical turnaround time of Java ED.S benchmark running on nine grid machines. Grey dots indicate benchmark did not pass verification because of machine unavailability. Large peak indicates a setup process of a fresh server on one of the grid machines.

overheads of currently implemented IPG services, which are likely to improve over time. The experiments, however, demonstrate the use of NGB to measure grid characteristics and as a pathfinding tool for grid application developers.

One important aspect, programmability of the IPG, was found to be in need of some improvement. Unexpected file clobbering by GridFTP, absence of third-party copying, and lack of functionality of its command line interface (no file renaming) leads to cumbersome programming. The exit status of jobs executed through g lobusrun cannot be communicated to the calling process, other than through explicit capture and file transfer by the programmer. globus_url_copy requires explicit knowledge of remote absolute paths, which hampers portability. Execution of Unix commands through globusrun requires knowledge of the paths to these commands on remote machines, which also hampers portability.

\section{Reîerences}

[1] R.M. Badia, J. Labrata, R. Sirvent, J.M. Pérez, J.M. Cela, and R. Grima. Programming Grid Applications with Grid Superscalar. http://www.zib.de/ggf/apps/meetings/ggf8/badial.pdf

[2] G. Chun, H. Dail, H. Casanova, A. Snavely. Benchmark Probes for Grid Assessment. UCSD Technical Report CS2003-0760, University of California, San Diego, CA, 2003.
[3] M.A. Frumkin, R.F. Van der Wijngaart. NAS Grid Benchmarks: A Tool for Grid Space Exploration. Cluster Computing Vol. 5, No. 3, pp.- 247-255, 2002.

[4] M.A. Frumkin, R. Hood. Using Grid Benchmarks for Dynamic Scheduling of Grid Applications. Proceedings of the 15th IASTED International Conference on Parallel and Distributed Computing and Systems (PDCS'2003), Marina Del Rey, USA, Nov. 3-5, 2003, p. 31-36, NAS Technical report NAS-03-015.

[5] W.E. Johnston, D. Gannon, B. Nitzberg. Grids as Production Computing Environments: The Engineering Aspects of NASA's Information Power Grid. Proc. $8^{\text {th }}$ IEEE IntI. Symp. High Performance Distributed Computing, Redondo Beach, CA, August 1999.

[6] A. Snavely, G. Chun, H. Casanova, R. Van der Wijngaart, M.A. Frumkin. Benchmarks for Grid computing: A Review of Ongoing Efforts and Future Directions. SIGMETRICS Performance Evaluation Review (PER), Vol. 30, No. 4, March 2003.

[7] R.F. Van der Wijngaart, M.A. Frumkin. NAS Grid Benchmarks Version 1.0. NAS Technical Report NAS02-005, NASA Ames Research Center, Moffett Field, $\mathrm{CA}, 2002$.

[8] R. Wolski, N.T. Spring, J. Hayes. The Network Weather Service: A Distributed Resource Performance Forecasting Service for Metacomputing. J. Future Generation Computing Systems, 1999.

[9] M. Zangrilli, B.B. Lowekamp. Comparing Passive Actrior Monitoring of Crid Application Traffic with Active Probes. SIGMETRICS Performance Evaluation Review (PER), Vol. 30, No. 4, March 2003.

[10] Globus Heartbeat Monitor. www.globus.org/hbm/.

[11] Globus Toolkit. www.globus.org.

[12] GRIDBench. www2.cs.ucy.ac.cy/georget'gridb/gridb.html.

[13] NASA's Information Dower Grid. www.ipg.nasa.gov.

[14] NAS Grid benchmarks. www.nas.nasa.gov/Software/NPB. 